\title{
The construction and validation of the Severe Asthma Questionnaire
}

\author{
Michael E. Hyland ${ }^{1,2}$, Rupert C. Jones ${ }^{2,3}$, Joseph W. Lanario ${ }^{1,2}$ and \\ Matthew Masoli ${ }^{2}$ \\ Affiliations: \\ ${ }^{1}$ School of Psychology, University of Plymouth, Plymouth, UK. \\ ${ }^{2}$ University Hospitals Plymouth NHS Trust, Plymouth, UK. \\ ${ }^{3}$ Faculty of Medicine and Dentistry, University of Plymouth, Plymouth, UK. \\ Correspondence: \\ Michael E. Hyland, School of Psychology, University of Plymouth, Plymouth, PL4 8AA, UK. \\ E-mail: mhylandaplymouth.ac.uk
}

@ERSpublications

Validating a new severe asthma-specific quality of life questionnaire, the SAQ

http://ow.ly/sSNW30k3Pbu

Cite this article as: Hyland ME, Jones RC, Lanario JW, et al. The construction and validation of the Severe Asthma Questionnaire. Eur Respir J 2018; 52: 1800618 [https://doi.org/10.1183/13993003.00618-2018].

ABSTRACT The US Food and Drug Administration's procedure for scale validation requires a documented stepwise process of qualitative and quantitative data. The aim of this paper is to provide final quantitative validating data for the Severe Asthma Questionnaire (SAQ).

The SAQ, Asthma Control Test, Mini Asthma Quality of Life Questionnaire and EQ-5D-5L were completed by 160 patients attending a severe asthma clinic; 51 patients completed the SAQ on two occasions for test-retest reliability analysis. The SAQ produces two scores, a SAQ score based on the average of 16 items and a SAQ-global score from a single 100-point global quality of life scale.

Construct validity was demonstrated by factor analysis of the 16 items, convergent validity by correlations of $>0.6$ between the SAQ, SAQ-global and other questionnaires, and discriminant validity by the ability of the SAQ and SAQ-global to distinguish between different treatment levels. Test-retest reliability (intra-class correlation) was 0.93 for the SAQ and 0.93 for the SAQ-global, and the alpha coefficient for the SAQ was 0.93 .

The SAQ was developed using recommended qualitative and quantitative procedures for scale development, and can be used to gain insight into patients' perceptions of how severe asthma and its treatment affects their lives.

This article has supplementary material available from erj.ersjournals.com

Received: March 312018 | Accepted after revision: May 112018

Copyright $\odot$ ERS 2018. This version is distributed under the terms of the Creative Commons Attribution Non-Commercial Licence 4.0. 


\section{Introduction}

Patients with severe and difficult-to-treat asthma comprise a small proportion (5-10\%) of all asthmatic patients, yet are responsible for a disproportionate degree of asthma morbidity and costs [1,2]. Quality of life assessment forms an essential part of asthma assessment because measures such as respiratory symptoms and lung function may not convey the true limitations caused by the disease from the patient's perspective [3]. Compared to patients with mild and moderate asthma, patients with severe asthma have additional quality of life deficits caused by comorbidities, higher treatment burden (particularly oral corticosteroids) and multiple and more severe exacerbations that disrupt the lives of patients and their relatives [4].

The US Food and Drug Administration (FDA) published recommendations for validating Patient Reported Outcome Measures (PROMs) in 2009 [5]. The US FDA recommends a stepwise process of validation: first, content validity is established by documented qualitative research; second, construct and other validity is established through quantitative methods.

The three most commonly used asthma-specific health-related quality of life scales [6-8] were published prior to the US FDA's 2009 guidelines and include symptom items that are perceived negatively in this context by patients [9]. These and other published asthma scales fail to assess quality of life deficits specific to severe asthma [10]. Thus, although existing scales are valid in terms of earlier definitions of validity [11, 12] or for mild and moderate asthma, they are not valid in terms of the US FDA's 2009 guidelines when used in people with severe asthma.

The qualitative stage of the development and content validation of the Severe Asthma Questionnaire (SAQ) has been reported in two studies [10, 13]. The first study [10] showed that in addition to deficits experienced in mild and moderate asthma, those with severe asthma also had problems caused primarily by two factors. First, the side effects of medication produced a variety of problems including mood changes, changes in self-perception, problems with eating, sleep disturbance and changes in appearance. Second, exacerbations (in particular those leading to hospitalisation) created problems for the patient and the wider family.

Using our findings from the first study we drafted a severe asthma-specific questionnaire, and subsequently conducted four focus groups in which patients with severe asthma provided iterative changes to the design and content of the draft questionnaire [13]. The aim of this second phase was to ensure that the questionnaire was able to correctly capture the kind of information patients felt important in relation to their quality of life. Patients defined the response scale, combined two items, split one item to assess different impacts on family lives, advised on recall period and optimised the wording of individual items. In addition, patients expressed a desire to provide an overall assessment of their quality of life (supplementary material).

The SAQ comprises 16 questions about different aspects of life and a global question that assesses their quality of life overall. The SMOG (simple measure of gobbledegook) grade [14] for the SAQ is 5.9, indicating suitability for a reading age of at least 11-12 years. The unweighted aggregation of the 16 questions produces a score similar in methodology to other quality of life questionnaires. The additional global question is used for two reasons. The first is because patients express a strong preference for providing an overall score in contrast to those requiring differentiation between different aspects of life. The second reason is psychological. Patients make judgements, including judgements about their lives, using either one or a combination of two cognitive processes, called System 1 and System 2. System 1 judgements are fast and automatic, and the process is unavailable to consciousness. System 2 judgements are slow and deliberative, and people can introspect the process [15]. A patient's response to a question in clinic of "how are you?" will be influenced primarily by a System 1 judgement. Using two types of measure, the SAQ score based on the 16 items and the SAQ-global score, provides a way of capturing these different kinds of judgement [16]. Single-item scales are less preferred in clinical trials because the use of multiple items reduces error variance.

The aim of this study was to provide the final stage of validation required by the US FDA, i.e. a quantitative study that establishes construct and other types of validity.

\section{Method}

\section{Participants}

Patients diagnosed with severe asthma as defined by the international consensus statement from the European Respiratory Society and American Thoracic Society [1] aged $\geqslant 16$ years of age who attended the Plymouth severe asthma service were invited to participate. Those with significant other conditions contributing to their respiratory symptoms, e.g. lung cancer, heart failure or severe chronic obstructive pulmonary disease, were excluded. 


\section{Questionnaires}

The SAQ comprises 16 items, with response options on a seven-point scale averaged to produce the SAQ score (scores 1-7) and a 100-point Borg-type scale [17] producing the SAQ-global score (scores 0-100). Quantifiers are indicated against all seven of the SAQ response options. A Borg scale is a category rating scale with quantifiers at either end and additional empirically placed quantifiers along the categories of the scale. The SAQ-global was adapted from an existing global quality of life scale [18] (more information is available from www.saq.org.uk).

The Mini Asthma Quality of Life Questionnaire (MiniAQLQ) [7] comprises 15 items with response options on a seven-point scale with responses averaged (scores 1-7).

The Asthma Control Test (ACT) [19] comprises five symptom and medication items (five response options per item) totalled to produce an asthma control score.

EQ-5D-5L comprises five quality of life items (five response options per item), which were averaged to produce an EQ-5D-5L score and the EQ-5D-VAS, which is a 100-point visual analogue scale (scores 1 100) $[20]$.

Criteria for missing data are shown in the supplementary material.

Clinical data

Clinical data collected were spirometry (forced expiratory volume in $1 \mathrm{~s}$ (FEV1)), prednisolone dose $\left(\mathrm{mg} \cdot \mathrm{day}^{-1}\right)$, number of severe exacerbations in the last 12 months requiring systemic steroids, Global Initiative for Asthma (GINA) severity, body mass index (BMI) and estimated cumulative oral corticosteroid (OCS) dose per year. The latter was calculated by multiplying the participant's maintenance steroid dose by 365 days, and adding an estimate of OCS use per exacerbation in the previous 12 months. Based on British Thoracic Society and GINA guidance, one exacerbation was judged to comprise prednisolone $40 \mathrm{mg} \cdot \mathrm{day}^{-1}$ multiplied by 7 days, which equates to $280 \mathrm{mg}$ of prednisolone per exacerbation $[21,22]$.

\section{Procedure}

Patients were recruited to the validation study or the reliability study or both studies. Questionnaires were completed either at home (postal return of questionnaire) or in clinic. Participants' data were included in an analysis of test-retest reliability if their ACT scores did not change by the minimum clinically important difference of $\geqslant 3$ points and they reported stable asthma (see supplementary material, supplementary figure E1).

\section{Analysis}

Analysis was on an intention-to-treat basis. Convergent validity between questionnaires was established using Pearson correlations. Groups of patients were identified by 1) level of maintenance dose of OCS $\left(\mathrm{mg} \mathrm{day}^{-1}\right)$ and 2$)$ estimated cumulative OCS dose per year. Differences between groups (discriminant validity) were tested by one-way ANOVA and where significant followed by least significant difference (LSD) post hoc tests. Construct validity was tested by exploratory factor analysis using principal axis factoring following recommended practice $[23,24]$. Evidence that the items could be aggregated into a single scale score was tested by examining whether the scree test indicated a unifactorial solution and whether items loaded significantly $(>0.3)$ on the first factor. Test-retest reliability was calculated by intra-class correlations. Internal consistency was calculated by Cronbach's alpha coefficient. All analyses were conducted using IBM SPSS statistics version 24 (Chicago, IL, USA).

\section{Ethical approval}

The study was approved by the University Hospitals Plymouth NHS Trust and Research Ethics Committee/Health Research Authority (ethical approval number 16/NE/0188, IRAS ID: 207601). All patients provided informed written consent.

\section{Results}

For the validation study, 260 participants were invited to participate: 20 declined to participate, 54 failed to return questionnaires by post and 26 failed to attend their clinic appointment and provide written informed consent, leaving 160 participants. For the test-retest reliability study, 115 patients were invited to participate, of whom 10 declined. Of the 105 who consented for the test-retest reliability study, 67 returned questionnaires and 16 patients were excluded because their ACT score had changed by $\geqslant 3$ points, leaving 51 participants (37 female) (supplementary figure E1). One patient at GINA step 2 was enrolled in the study in error; they did not meet the criteria for severe asthma but were at higher risk. Their data have 


\begin{tabular}{|c|c|c|c|}
\hline & All & GINA step 4 & GINA step 5 \\
\hline Subjects n & $160^{\#}$ & 100 & 59 \\
\hline Female & $107(66)$ & 67 (67) & 40 (67) \\
\hline Age years & $51(16-78)$ & $49(16-74)$ & $55(25-78)$ \\
\hline FEV1 L & $2.0(0.7-4.4)$ & $2.2(0.72-4.3)$ & $1.8(0.7-3.6)$ \\
\hline FEV $1 \%$ predicted & $72(28-137)$ & $75(28-137)$ & 65 (34-107) \\
\hline Caucasian \% & 98 & & \\
\hline$B M I \mathbf{k g} \cdot \mathrm{m}^{-2}$ & $31.8(18.2-58.3)$ & $31.0(19.0-58.3)$ & $33.3(20.7-57.9)$ \\
\hline $\begin{array}{l}\text { Median ICS dose BDP equivalent } \mu \mathrm{g} \cdot \mathrm{day}^{-1} \\
\text { Taking biologics }\end{array}$ & $1600(400-4000)$ & $1600(800-4000)$ & $2000(1000-4000)$ \\
\hline Omalizumab & $34(21)$ & & \\
\hline Mepolizumab & $4(3)$ & & \\
\hline
\end{tabular}

Data are presented as $\mathrm{n}$ (range) or $\mathrm{n}(\%)$, unless otherwise stated. GINA: Global Initiative for Asthma; FEV1: forced expiratory volume in $1 \mathrm{~s}$; BMI: body mass index; ICS: inhaled corticosteroid; BDP: beclomethasone dipropionate. \# : one patient at GINA step 2 was included erroneously but included in the intention-to-treat analysis, see Results section.

been included in the analysis because this study used intention-to-treat criteria. Participant demographics are displayed in table 1.

Of the 160 patients who participated in the validation study, 154 (96\%) completed the SAQ, $146(91 \%)$ the MiniAQLQ and 159 (99\%) the ACT. Of the 100 patients asked to complete the EQ-5D-5L, 96 (96\%) complied.

Table 2 shows the correlations between the different questionnaires. All correlations were $>0.60$, showing convergence between the different questionnaires. The correlations between all questionnaires, BMI and FEV1 \% predicted are also shown.

Table 3 shows the mean \pm SD of responses to the 16 items of the SAQ. Absence of floor or ceiling effects is indicated by no SAQ item having a standard deviation less than one point away from an end point. In addition, participants used the full range of response options for all items with some using every option between 1 and 7 for all items (supplementary table E1). These results show that no item should be rejected on the basis of poor distribution. The SAQ scores ranged from 5 (extremely bad quality of life) to 100 (perfect quality of life) (supplementary table E2).

When testing whether it is valid to aggregate the 16 items into a single scale score, exploratory factor analysis of the SAQ revealed a one-factor solution using the scree test (the first four eigenvalues were 9.91, $1.11,0.92$ and 0.72 ) accounting for $60 \%$ of the variance. The factor scores are shown in table 3 . All factor scores are $>0.6$, showing that despite difference in content all items were related to the same latent variable. Cronach's alpha coefficient was 0.96 . Test-retest reliability as measured by intra-class correlation was 0.93 (95\% confidence interval (CI) 0.87-0.96) for the SAQ and 0.93 (95\% CI 0.86-0.96) for the SAQ-global, showing that the SAQ is a reliable scale. Test-retest reliability was also calculated individually for each item of the SAQ (supplementary table E3).

TABLE 2 Pearson's correlations between all six scales, BMI and FEV $1 \%$ predicted

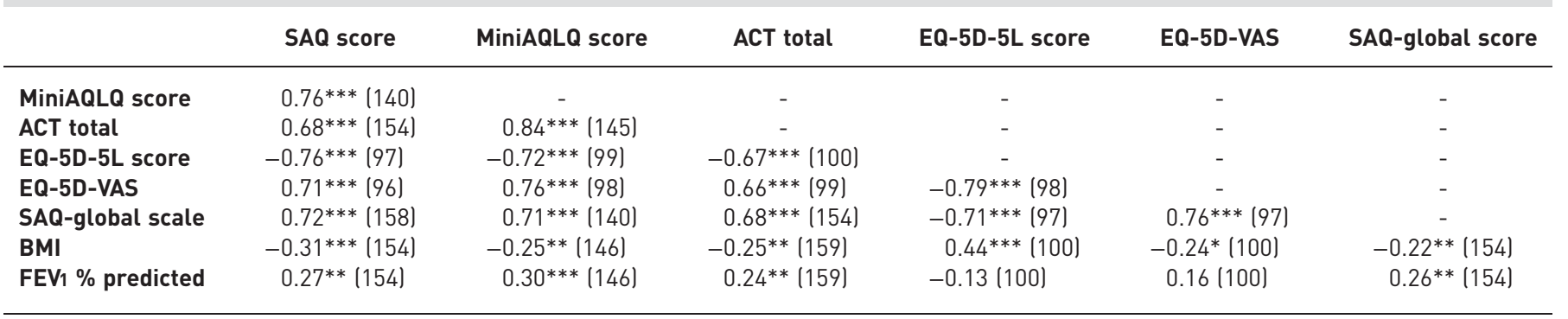

Data are presented as correlation coefficient (n). SAQ: Severe Asthma Questionnaire; MiniAQLQ: Mini Asthma Quality of Life Questionnaire; ACT: Asthma Control Test; VAS: visual analogue scale; BMI: body mass index; FEV1: forced expiratory volume in $1 \mathrm{~s} .{ }^{*}: p<0.05,{ }^{* *}: \mathrm{p}<0.01$, $* * *: p<0.001$. 
TABLE 3 Mean $\pm S D$, factor loadings and the percentage of participants responding "very difficult" or "very, very difficult" to each of the 16 items of the Severe Asthma Questionnaire

Item

$\begin{array}{ccc}\text { Mean } \pm s D^{\#} & \begin{array}{c}\text { Patients responding } \\ \text { "very difficult" or } \\ \text { "very, very difficult" \% }\end{array} & \begin{array}{c}\text { Factor } \\ \text { loading }\end{array} \\ & \end{array}$

1. My social life. For example, visiting friends, walking with friends, talking with friends, going to bars/restaurants, and parties.

2. My personal life. For example, washing, dressing, looking after myself, love life.

3. My leisure activities. For example, walking for pleasure, sports, exercise, travelling, taking vacations.

4. My jobs around the house. For example, housework, shopping, home maintenance, gardening.

5. My work or education. For example, missing days, can't do all I want to do.

6. My family life - how it affects me. For example, caring for children, family responsibilities.

7. My family life - how it affects others. For example, others taking time off work, problems with childcare, family members becoming upset.

8. Depression. For example, feeling sad, fed up, blue.

9. Irritable. For example, snap at people, get angrier than I should.

10. Anxiety in general. For example, worry about things, always on edge.

$4.11 \pm 1.94 \quad 25.2$

25.2

0.88

$4.98 \pm 1.98$

15.0

$3.54 \pm 1.95 \quad 37.5$

$3.96 \pm 1.92$

27.2

0.84

$4.78 \pm 2.64$

25.7

0.88

$4.59 \pm 2$

0.6

$4.74 \pm 2.1$

19.6

11. Worry that asthma may get worse. For example, medicines no longer help, more frequent attacks.

12. Worry about long-term side effects of medicines. For example, worry about cataracts, diabetes, bone fracture.

13. Getting tired. For example, feeling tired for no reason, waking in the morning feeling tired.

$\begin{array}{lll}3.92 \pm 2.12 & 33.8 & 0.66\end{array}$

$3.16 \pm 1.9 \quad 43.3$

0.79

14. Problems at night. For example, difficulty going to sleep, being woken very easily, waking often at night.

15. The way I look. For example, my weight, my skin bruises easily, using medicines in public, other people judging me

16. Problems with food. For example, I find I get very hungry, I just can't stop eating, stomach problems (e.g. pain, bloating, etc.)

\#: interpretation of mean in relation to quality of life, 1=very, very difficult (worst possible); 2=very difficult; 3=difficult; 4=moderately difficult; $5=$ slightly difficult; $6=$ very slightly difficult (just noticeable); $7=$ no problem.

Table 4 shows the mean score values for each of the six scales as a function of four groups of patients stratified by maintenance prednisolone dose: no maintenance prednisolone, $1-9 \mathrm{mg} \cdot \mathrm{day}^{-1}, 10 \mathrm{mg} \cdot \mathrm{day}^{-1}$ and $>10 \mathrm{mg} \cdot \mathrm{day}^{-1}$ (ranges were selected to achieve groups of approximately equal size). All scales were significantly different across the four groups $(p<0.001)$, showing that the SAQ can discriminate between groups that are theoretically predicted to be different based on asthma severity. To compare the discrimination of different questionnaires between the $10 \mathrm{mg} \cdot \mathrm{day}^{-1}$ and $>10 \mathrm{mg} \cdot \mathrm{day}^{-1}$ groups as a function of maintenance dose, we conducted LSD tests. Significant differences were found for the SAQ score $(\mathrm{p}=0.01)$, SAQ-global score $(\mathrm{p}=0.01)$ and $\mathrm{EQ}-5 \mathrm{D}$-VAS score $(\mathrm{p}=0.02)$, but not the MiniAQLQ score $(\mathrm{p}=0.13)$, ACT score $(\mathrm{p}=0.34)$ or EQ-5D-5L score $(\mathrm{p}=0.23)$. With regard to other paired comparisons, there was a pattern of poorer outcome with increasing dose of OCS except for the comparison between those on zero dose and those on 1-9 mg.day ${ }^{-1}$. For these comparisons (using LSD tests), the patients taking 1-9 mg.day ${ }^{-1}$ had better quality of life than those on zero dose according to the MiniAQLQ $(\mathrm{p}=0.008)$, ACT $(\mathrm{p}=0.001)$ and SAQ $(\mathrm{p}=0.017)$ but not the SAQ-global $(\mathrm{p}=0.19)$, EQ-5D-5L $(\mathrm{p}=0.2)$ or EQ-5D-VAS ( $p=0.4)$. Because the $1-9 \mathrm{mg} \cdot \mathrm{day}^{-1}$ group had better quality of life than the zero dose group, we examined possible differences in biologic prescription between the four groups. In the zero dose group, 17 out of $101(17 \%)$ were on biologics, in comparison to nine out of $17(53 \%)$ in the $1-9 \mathrm{mg} \cdot$ day $^{-1}$ group, six out of $20(30 \%)$ in the $10 \mathrm{mg} \cdot \mathrm{day}^{-1}$ group and six out of $22(27 \%)$ in the $>10 \mathrm{mg}^{-d a y}{ }^{-1}$ group. Figure 1 provides a visual comparison of the SAQ and MiniAQLQ as a function of maintenance prednisolone dose.

Participants were allocated to estimated annual cumulative OCS dose groups: 0-1119 mg.year ${ }^{-1}$, 1120-1460 mg.year ${ }^{-1}, 1461-3650 \mathrm{mg} \cdot \mathrm{year}^{-1}, 3651-6595 \mathrm{mg} \cdot \mathrm{year}^{-1}$ and $>6595 \mathrm{mg} \cdot \mathrm{year}^{-1}$. These doses were chosen to be clinically relevant, e.g. $1120 \mathrm{mg} \cdot$ year $^{-1}$ equates to approximately four courses of OCS, $3650 \mathrm{mg} \cdot \mathrm{year}^{-1}$ to $\sim 10 \mathrm{mg} \cdot \mathrm{day}^{-1}$ prednisolone and $>6595 \mathrm{mg} \cdot \mathrm{year}^{-1}$ to $\sim 20 \mathrm{mg} \cdot \mathrm{day}^{-1}$ prednisolone. Table 5 shows the mean scores for these five groups for each of the six scales. Figure 2 provides a visual comparison of the SAQ and MiniAQLQ as a function of cumulative prednisolone dose. 
TABLE 4 Mean, confidence intervals and n-values for questionnaire scores at different levels of prednisolone dose

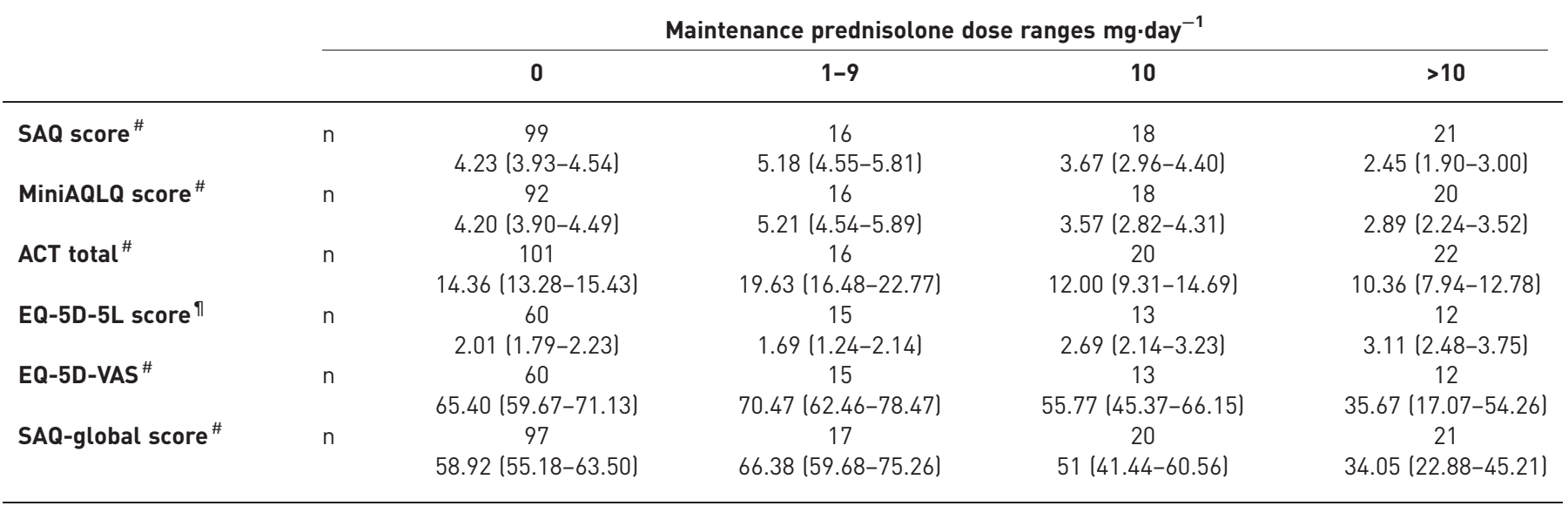

Data are presented as mean $(95 \% \mathrm{Cl})$, unless otherwise stated. SAQ: Severe Asthma Questionnaire; MiniAQLQ: Mini Asthma Quality of Life Questionnaire; ACT: Asthma Control Test; VAS: visual analogue scale. ${ }^{\#}$ : a lower score indicates low quality of life/health; ${ }^{\text {?: }}$ a high score indicates poor health.

One-way ANOVA showed a significant difference $(\mathrm{p}<0.001)$ between the five estimated cumulative OCS dose groups for all questionnaires. In order to carry out post hoc tests that are equivalent to those of table 4 , we combined the two groups with the highest burden, i.e. those on 3651-6595 mg.year ${ }^{-1}$ and those on $>6595 \mathrm{mg} \cdot \mathrm{year}^{-1}$. ANOVA was repeated on the four groups (all questionnaires were significant at $\mathrm{p}<0.001)$. Significant differences were found between those on 1461-3650 mg.year ${ }^{-1}$ and $>3650 \mathrm{mg} \cdot \mathrm{year}^{-1}$ for the SAQ score $(p<0.001)$, MiniAQLQ score $(p<0.01)$, ACT total $(p=0.004)$, EQ-5D-5L score $(\mathrm{p}=0.003)$, SAQ-global score $(\mathrm{p}=0.006)$ and EQ-5D-VAS $(\mathrm{p}=0.04)$.

\section{Discussion}

The US FDA's guidance for valid questionnaire construction [5] requires documentation of a qualitative stage of research followed by a quantitative stage. The SAQ was developed on the basis of qualitative research reported elsewhere $[10,13]$. In this paper we provide details of the quantitative stage.

People with severe asthma experience difficulty in a number of different domains of life experience, some of which are not experienced by those with mild and moderate asthma. For example, $28 \%$ felt that irritability, a side effect of OCS, made life either very difficult or very, very difficult, and $27 \%$ experienced this level of difficulty with food. "Problems at night" were reported by $39 \%$ to make life at least very difficult; the sleep disturbance can be caused by either asthma symptoms (which cause waking) or the side effects of OCS (which cause difficulty getting to sleep, waking and additional problems during the night). Two family items are included in the SAQ as a result of the earlier qualitative research [13]: 20\% of

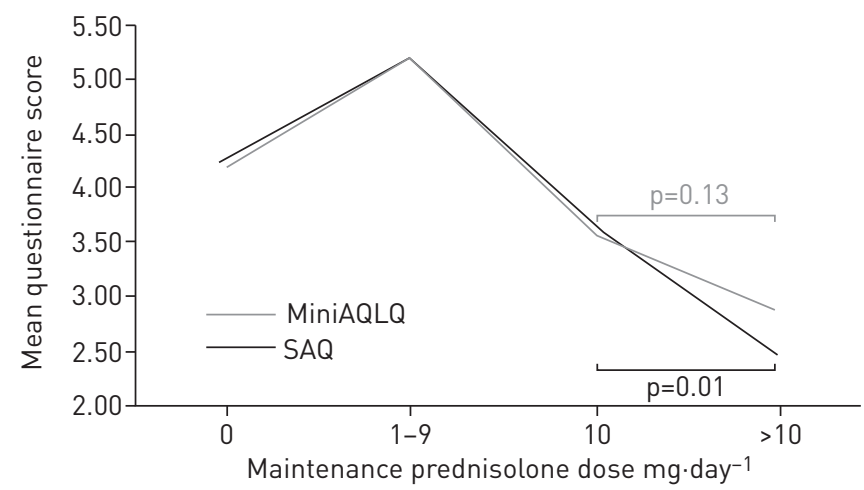

FIGURE 1 Mean Severe Asthma Questionnaire (SAQ) and Mini Asthma Quality Of Life Questionnaire (MiniAQLQ) scores as a function of maintenance prednisolone dose $\left(\mathrm{mg} \cdot \mathrm{day}^{-1}\right)$. When comparing $10 \mathrm{mg}^{\mathrm{d}} \mathrm{day}{ }^{-1}$ to $>10 \mathrm{mg} \cdot$ day $^{-1}$ of maintenance prednisolone use, the $S A Q$ detects a statistically significant difference in mean questionnaire score, but the MiniAQLQ does not. 
TABLE 5 Mean, confidence intervals and n-values for questionnaire scores at different levels of estimated annual cumulative oral corticosteroid dose

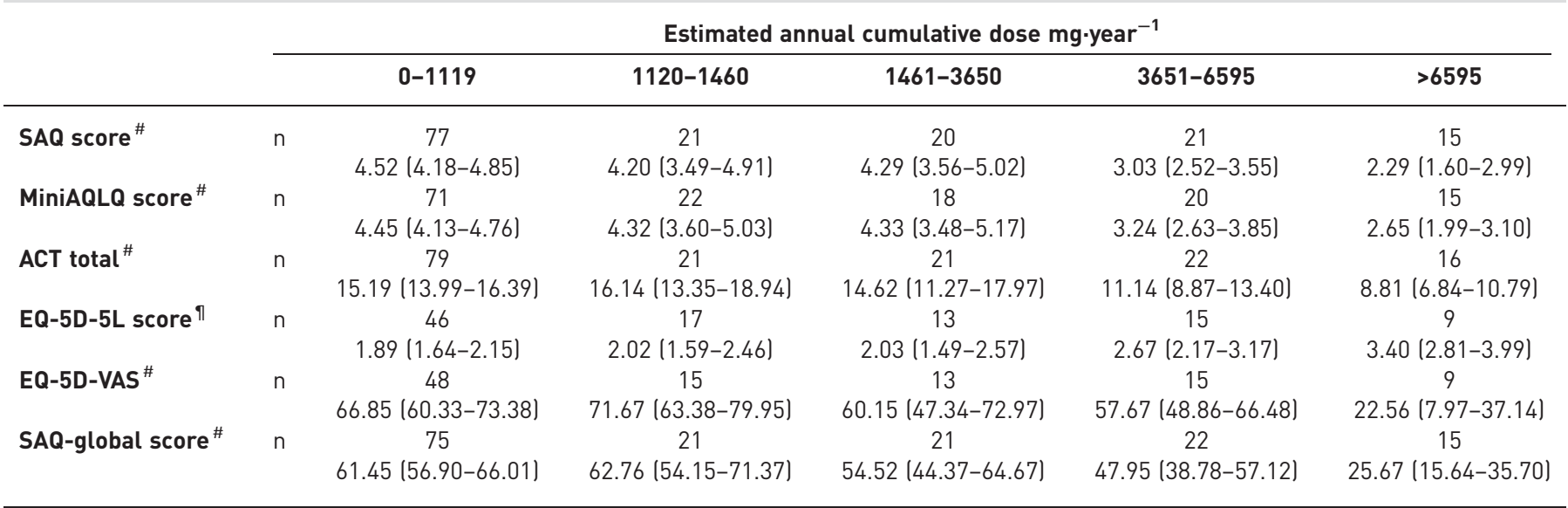

Data are presented as mean (95\% CI), unless otherwise stated. SAQ: Severe Asthma Questionnaire; MiniAQLQ: Mini Asthma Quality of Life Questionnaire; ACT: Asthma Control Test; VAS: visual analogue scale. ${ }^{\#}$ : a lower score indicates low quality of life/health; " : a high score indicates poor health.

patients rated their family lives as at least very difficult for themselves, and $18 \%$ rated it at least very difficult for other family members. These results confirm the earlier qualitative research that severe asthma impacts family life for not only the patient but also the patient's family. Our descriptive results show that people with severe asthma can experience nontrivial disutility in domains of experience which are not included in questionnaires designed for mild and moderate asthma, with particularly high disutility recorded for "the way I look", "getting tired" and the two items assessing different worries about medication.

Although some people with severe asthma experience very poor quality of life, this is not a universal experience. For example, although $32 \%$ of people in this study felt they had moderately bad quality of life or worse, $24 \%$ felt they had good quality of life or better. Although some of this difference can be explained by severity and treatment differences (those with greater OCS burden report poorer quality of life), the relationship between severity, treatment and quality of life is complex.

In addition to providing descriptive data on people with severe asthma, the data provide construct validity for the SAQ. Statistical analysis showed that all 16 items could be aggregated into a single score and that no item should be rejected on purely statistical grounds. The intra-class test-retest reliability of the SAQ and SAQ-global of 0.93 compares well with other questionnaires, e.g. 0.83 for the MiniAQLQ [7]. The SAQ score and the SAQ-global score correlate with other asthma and generic scales, thereby providing convergent validity. The SAQ score discriminates between groups of patients defined by maintenance prednisolone dose and defined by estimated cumulative OCS burden per year, thereby providing discriminant validity. There is a trend for the SAQ to demonstrate greater quality of life impairment with

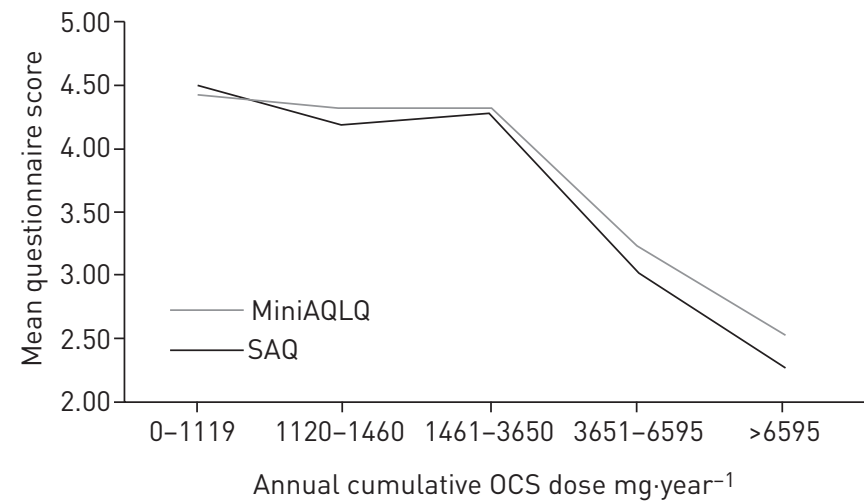

FIGURE 2 Mean Severe Asthma Questionnaire (SAQ) and Mini Asthma Quality Of Life Questionnaire (MiniAQLQ) scores as a function of estimated annual cumulative oral corticosteroid (OCS) dose. 
increasing OCS burden compared to the MiniAQLQ but the study is underpowered to make statistical comparisons between scales.

All outcome measures showed a trend towards poorer quality of life with greater doses of OCS, except for the comparison between those on no prednisolone versus those prescribed $1-9 \mathrm{mg} \cdot \mathrm{day}^{-1}$. We do not know why the 1-9 mg.day ${ }^{-1}$ group had better quality of life than those without a maintenance dose, but it may be due to the higher use of biologics in the $1-9 \mathrm{mg}^{-d_{a y}}{ }^{-1}$ group. Further studies are needed to clarify the complex relationship between biologics and OCS on quality of life versus asthma symptoms [25].

The SAQ differs from other asthma-specific scales in that it includes a single-item 100-point scale of global quality of life, the SAQ-global, which is included because of patient request. In this respect the SAQ is similar to the EQ-5D, in which there is also a single-item 100-point scale, the EQ-5D-VAS. The SAQ-global is a 0-100 Borg-type scale adapted from another scale [18], and research shows the use of additional quantifiers is more reliable than the format used in the EQ-5D-VAS [15] in which only the end points have quantifiers. With the exception of the EQ-5D and EQ-5D-VAS, all questionnaires show weak to moderate correlations with lung function, indicating that lung function has a modest causal effect on these measures. The absence of a significant correlation for the EQ-5D and EQ-5D-VAS suggests that these two measures might not be valid for assessing the quality of life in patients with severe asthma.

Multiple-item scales often have better reliability than single-item scales for statistical reasons. Consistent with other research showing Borg scales to be more reliable than visual analogue scales [18], our data show that the SAQ-global is highly reliable and as reliable as the SAQ score. The SAQ-global also correlates well with other scales. Comparison between the EQ-5D and EQ-5D-VAS suggests that the EQ-5D-VAS measures a broader concept than the EQ-5D [16], and the same difference may apply to the SAQ score and SAQ-global. Additionally, response to these two different parts of the SAQ may reflect different cognitive processes, a fast automatic process for the SAQ-global and a slow deliberate process for the SAQ score [15]. Our study shows that ratings of "good quality of life" on the SAQ-global (i.e. those in the 70-79 range) equate to "makes my life slightly difficult" (i.e. a mean score of 4.8 on the SAQ; supplementary table E2). This demonstrates that the way a question is asked affects the patient's response. Both types of measure can be useful, but it is likely that the SAQ-global provides a value more consistent with patients' immediate response when clinicians ask about their asthma.

\section{Limitations}

The estimated cumulative OCS dose might be an underestimate of OCS burden because patients with severe asthma often require a longer course of OCS for an exacerbation than the 7 days used in our calculation. The use of biologics was not considered in this analysis and could affect quality of life assessments. The study was carried out in the South West of the UK, and although the demographic data of our study population are not dissimilar to the UK demographic data presented within the UK severe asthma registry [26], the population was predominantly Caucasian and further validation in ethnically and culturally diverse populations is required. A reading age of at least age 11-12 years old is required to use the SAQ, but this is consistent with current literacy requirements for patient communication [27]. We used the 15-item MiniAQLQ rather than the 32-item AQLQ as a comparison scale to reduce questionnaire fatigue and because the MiniAQLQ is similar in length to the SAQ. Nine patients participating in this study also participated in an earlier qualitative work [13].

The SAQ is structured so that the global measure appears after the 16 context-specific items, and this order of presentation may have an effect. Research shows that prior exposure to a negative event (i.e. asking patients about difficulty in different contexts) will lead to more positive subsequent judgement [28, 29]. Further research is needed to explore how contextual factors, including the order of presentation of different questions, influences quality of life judgements.

\section{Conclusions}

The SAQ was designed to detect the impact of both asthma symptoms and treatment on quality of life and has been shown to be content valid in earlier studies $[10,13]$. This paper confirms the relevance of items based on the earlier studies, establishes the construct validity of the scale, and shows the scale to be reliable in a group of patients with severe asthma with different types of treatment. These data complete the validation procedures required by the US FDA. Longitudinal studies are required to provide further information about the scale, e.g. to establish sensitivity to change and the minimal clinically important difference in people with severe asthma. The SAQ is available for use in the British English version reported here and is available from the authors or online (www.saq.org.uk). Translations in other languages are required. In addition to providing a scale that is fit for purpose [10] for assessing health-related quality of life in clinical trials, the SAQ can also be used in clinical practice to alert 
clinicians to the problems experienced by patients, and to gain an initial insight into the patients' own perceptions of the impact on their lives of illness and its treatment.

Acknowledgements: We acknowledge Jill Pooler's early work in the project including help in preparing for and obtaining NHS and REC/HRA ethical approval. Mike Thomas provided helpful comments on an earlier draft. We would also like to thank the staff at Derriford Hospital's Chest Clinic for their assistance with this project.

Author contributions: All four authors contributed to the design of the study and all four authors contributed to the writing of the paper. M.E. Hyland wrote the first draft and was responsible for the analysis. M. Masoli and R.C. Jones were responsible for recruitment and data collection. J.W. Lanario collected the data and contributed to the analysis.

Conflict of interest: M.E. Hyland reports an unrestricted medical educational grant from Novartis and non-promotional grant from AstraZeneca during the conduct of the study, and speaker fees from Novartis and GlaxoSmithKline outside the submitted work. R.C. Jones reports an unrestricted medical educational grant from Novartis and non-promotional grant from AstraZeneca, during the conduct of the study; and personal fees from GlaxoSmithKline, AstraZeneca, Boehringer-Ingelheim, Chiesi, Cipla, Novartis and Pfizer outside the submitted work. J.W. Lanario reports an unrestricted medical educational grant from Novartis and non-promotional grant from AstraZeneca during the conduct of the study and speaker fees from Novartis outside the submitted work. M. Masoli reports unrestricted non-promotional grants from Novartis and AstraZeneca, who supported the initial study of patient interviews and questionnaire development. The SAQ validation study has been supported through Plymouth Hospitals NHS Trust research fund. M. Masoli has received speaker fees from Novartis and Astra Zeneca outside the submitted work.

Support statement: Funding provided by AstraZeneca (non-promotional grant). R.C. Jones and J.W. Lanario were supported by the National Institute for Health Research (NIHR) Collaboration for Leadership in Applied Health Research and Care South West Peninsula (NIHR CLAHRC South West Peninsula). The views expressed are those of the authors and not necessarily those of the NHS, the NIHR or the Department of Health and Social Care. Funding information for this article has been deposited with the Crossref Funder Registry.

\section{References}

1 Chung KF, Wenzel SE, Brozek JL, et al. International ERS/ATS guidelines on definition, evaluation and treatment of severe asthma. Eur Respir J 2013; 43: 343-373.

2 O'Neill S, Sweeney J, Patterson CC, et al. The cost of treating severe refractory asthma in the UK: an economic analysis from the British Thoracic Society Difficult Asthma Registry. Thorax 2014; 70: 376-378.

3 Wilson SR, Rand CS, Cabana MD, et al. Asthma outcomes: quality of life. J Allergy Clin Immunol 2012; 129: S88-S123.

4 Sweeney J, Patterson CC, Menzies-Gow A, et al. Comorbidity in severe asthma requiring systemic corticosteroid therapy: cross-sectional data from the Optimum Patient Care Research Database and the British Thoracic Difficult Asthma Registry. Thorax 2016; 71: 339-346.

5 US Department of Health and Human Services Food and Drug Administration. Guidance for Industry: Patient-reported outcome measures: use in medical product development to support labeling claims. Silver Spring, US Food and Drug Administration, 2009.

6 Juniper EF, Guyatt GH, Ferrie PJ, et al. Measuring quality of life in asthma. Am Rev Respir Dis 1993; 147: 832-823.

7 Juniper EF, Guyatt GH, Cox FM, et al. Development and validation of the Mini Asthma Quality of Life Questionnaire. Eur Respir J 1999; 14: 32-38.

8 Jones PW, Quirk FH, Baveystock CM, et al. A self-complete measure of health status for chronic airflow limitation. Am Rev Respir Dis 1992; 145: 1321-1327.

9 Apfelbacher CJ, Jones CJ, Frew A, et al. Validity of three asthma-specific quality of life questionnaires: the patients' perspective. BMJ Open 2016; 6: e011793.

10 Hyland ME, Whalley B, Jones RC, et al. A qualitative study of the impact of severe asthma and its treatment showing that treatment burden is neglected in existing asthma assessment scales. Qual Life Res 2015; 24: 631-639.

11 Edwards MC, Slagle A, Rubright JD, et al. Fit for purpose and modern validity theory in clinical outcomes assessment. Qual Life Res 2017; in press.

12 Anastasi A. Psychological Testing. 3rd Edn. New York, Macmillan, 1968.

13 Hyland ME, Lanario JW, Pooler J, et al. How patient participation was used to develop a questionnaire that is fit for purpose for assessing quality of life in severe asthma. Health Qual Life Outcomes 2018; 16: 24.

14 Hedman AS. Using the SMOG formula to revise a health-related document. Am J Health Educ 2008; 39: 61-64.

15 Kahneman D. Thinking, Fast and Slow. New York, Macmillan, 2011.

16 Feng Y, Parkin D, Devlin NJ. Assessing the performance of the EQ-VAS in the NHS PROMs programme. Qual Life Res 2014; 23: 977-989.

17 Borg GA. Psychophysical bases of perceived exertion. Med Sci Sports Exerc 14: 377-381.

18 Hyland ME, Sodergren SC. Development of a new type of global quality of life scale, and comparison of performance and preference for 12 global scales. Qual Life Res 1996; 5: 469-480.

19 Nathan RA, Sorkness CA, Kosinski M, et al. Development of the asthma control test: a survey for assessing asthma control. J Allergy Clin Immunol 2004; 113: 59-65.

20 Herdman M, Gudex C, Lloyd A, et al. Development and preliminary testing of the new five-level version of EQ-5D (EQ-5D-5L). Qual Life Res 2011; 20: 1727-1736.

21 BTS/SIGN. SIGN 153. British Guideline on the Management of Asthma. www.britthoracic.org.uk/ documentlibrary/clinicalinformation/asthma/btssign-asthma-guideline-2016/ Date last updated: September 1, 2016. Date last accessed: April 27, 2018.

22 GINA. 2018 GINA Report, Global Strategy for Asthma Management and Prevention. http://ginasthma.org/ 2018-gina-report-global-strategy-for-asthma-management-and-prevention/ Date last updated: March 29, 2018. Date last accessed: April 27, 2018. 
23 Costello AB, Osborne JW. Best practices in exploratory factor analysis: four recommendations for getting the most from your analysis. Pract Assess Res Eval 2005; 10: 1-9.

24 Osborne JW. Best Practices in Exploratory Factor Analysis. North Charleston, CreateSpace Independent Publishing Platform, 2014.

25 Stucky BD, Sherbourne CD, Edelen MO, et al. Understanding asthma-specific quality of life: moving beyond asthma symptoms and severity. Eur Respir J 2015; 46: 680-687.

26 Heaney LG, Brightling CE, Menzies-Gow A, et al. Refractory asthma in the UK: cross-sectional findings from a UK multicentre registry. Thorax 2010; 65: 787-794.

27 Paasche-Orlow MK, Taylor HA, Brancati FL. Readability standards for informed-consent forms as compared with actual readability. N Engl J Med 2003; 348: 721-726.

28 Johnson JT. The knowledge of what might have been: affective and attributional consequences of near outcomes. Pers Soc Psychol Bull 1986; 12: 51-62.

29 Medvec VH, Madey SF, Gilovich T. When less is more: counterfactual thinking and satisfaction among Olympic medalists. J Pers Soc Psychol 1995; 69: 603. 